\title{
Management of irritable bowel syndrome in children
}

\author{
Juan R Malagelada, Carolina Malagelada
}

'Digestive System Research Unit, University Hospital Vall d'Hebron, Centro de Investigación Biomédica en Red de Enfermedades Hepáticas y Digestivas (Ciberehd), Department of Medicine, Universitat Autònoma de Barcelona, Barcelona, Spain

Article Info

\section{Article Notes}

Received: April 23, 2018

Accepted: June 28, 2018

\section{*Correspondence:}

Dr. Juan R Malagelada

Digestive System Research Unit, University Hospital Vall d'Hebron; Centro de Investigación Biomédica en Red de Enfermedades Hepáticas y Digestivas (Ciberehd); Department of Medicine, Universitat Autònoma de Barcelona, Barcelona, Spain

E-mail: juanrmalagelada@gmail.com

(c) 2018 Malagelada JR. This article is distributed under the terms of the Creative Commons Attribution 4.0 International License.

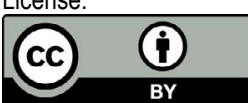

\section{Overview}

IBS is a clinical condition involving frequent ( $\geq 4$ days per month) and chronic ( $>2$ months) abdominal pain and stooling disturbance. Bloating is a common and highly annoying accompanying symptom. Diagnosis is established by applying symptom criteria elucidated through a series of medical expert pools (the "ROME" process I, II, III, and IV). Diagnostic criteria are quite similar for children and adults ${ }^{1}$. With regard to bowel movement disturbances, a constipation predominant pattern is more common in children than a diarrhea predominant pattern or a mixed, alternating constipation/diarrhea pattern $^{2-4}$. Pattern shifts over time are rather common ${ }^{3}$.

IBS disease mechanisms so far identified are multiple, varied and not always fully understood. Pathophysiological features of IBS that are supported by solid scientific evidence include visceral hypersensitivity, gastrointestinal dysmotility, gut inflammation, dietary triggers, intestinal dysbiosis and disturbances of the gut-brain axis. Thus, IBS may be considered pathogenetically a heterogeneous entity, as recently reviewed ${ }^{5}$. Familial aggregation is often noticeable but it is unclear whether genetic or environmental influences, including heightened awareness of abdominal sensations, are responsible.

IBS is quite common in children as well as in adults. A well conducted, albeit relatively older study estimated that $14 \%$ of US high school students and 6\% of middle school students suffer from symptoms consistent with IBS ${ }^{6}$. More recent data generally supports a very high prevalence of IBS in children ${ }^{2,4,7}$ and consequently a high economic burden ${ }^{8}$. In clinical practice, an IBS diagnosis is not infrequently applied rather loosely since IBS is defined exclusively by a relatively complex symptom formula without a verifiable biological marker. Therefore, clinicians sometimes apply the IBS label to any patient without evidence of "definite" organic disease who complains of non-acute abdominal pain combined with altered bowel movement pattern. As pointed out by Quigley \& Shanahan ${ }^{9}$, a medical condition such as IBS, that is exclusively defined by symptom criteria, may create an illusion of understanding and equate complaints with "disease". This aspect could be particularly relevant in children who may express symptoms vaguely and inconsistently. The real "intensity of complaints" may also be difficult to ascertain in this age group of patients. Stressful events affecting child and family as well as attitudes and environmental factors are important ${ }^{10,11}$. 
Prudent and carefully weighted application of diagnostic tests is in order. A "positive" IBS diagnosis based on symptom criteria should be attempted first, rather than an "exclusion" IBS diagnosis as traditionally undertaken because the latter implies much higher iatrogenic risks and/ or economic burden. However, some basic evaluations way be warranted if in doubt, if family pressures are significant, if prompt resolution is not achieved by application of first line therapeutic measures and, of course, if alarm clinical features are observed. The first commonly applied diagnostic examinations include blood test, stool check for parasites or blood, abdominal ultrasound exam and endoscopic evaluation of the gastrointestinal tract.

Clinicians evaluating a child with abdominal symptoms also need to pay attention to extraintestinal manifestations and comorbid conditions such as fatigue, headaches, urinary and musculoskeletal symptoms etc as shown by IBS adult studies $^{12}$. The coincidence of these various symptomatic expressions in the same individual suggests CNS participation distorting and integrating peripheral inputs leading to the concept of central sensitizing disorders that may include IBS. Indeed, children with chronic digestive symptoms show exaggerated brainstem responses to surrounding stimuli and inadequate parental behaviours appear to aggravate symptoms ${ }^{13}$. Conversely, seemingly spontaneous resolution of IBS is not uncommon ${ }^{14}$.

An IBS label in today's hyper-communicated society may also incorporate the conceptual elements of a "meme" spreading by contagion among clinicians, relatives or child-caring personnel ${ }^{15}$. Symptoms themselves may be potentially promoted and amplified by the child's entourage and even by medical personnel, eager to establish a diagnosis in a child with otherwise unexplained abdominal complaints. This mechanism could explain the increasing prevalence of IBS diagnosis in western societies both in children and adults.

\section{Useful pathophysiological features of IBS that may help guide appropriate therapy}

If considering treatment for IBS, it may be useful to attempt to ascertain the pathogenetic mechanism or, at least, the predominant pathophysiological disturbance operating in individual patients, because a mechanismbased approach probably improves therapeutic effectiveness ${ }^{5}$.

Diet composition, tolerance and therapeutic adjustments

Diet has the potential to act both as a symptom inductor and a therapeutic opportunity. It is self-evident that not all children tolerate equally the multiple components of a normal diet. Specific food allergies may manifest in a clinically obvious manner that leaves no doubt as to their pathogenetic role. However, more subtle forms of immune nutrient-cell interactions may be at the root of IBS-like symptoms ${ }^{16,17}$. The challenge is to establish a definite responsibility for specific food products in the genesis of symptoms when simple observation yields only equivocal answers. A number of commercial tests are nowadays available under the label "food intolerance tests". These include allergen-specific IgG reaction against various foods, electrodermal tests, sublingual or intradermal tests, cytotoxic assays and others. Although several of these tests have elicited interest among physicians and, particularly, among the general public they have not reached solid scientific approval and there are doubts as to whether test results truly reflect clinically relevant intolerance. Furthermore, there is mounting concern that unwarranted and radical diet restrictions may lead to nutritional imbalances including accumulation of metallic elemental toxicants (i.e. mercury) in blood and tissues ${ }^{18}$.

Non-allergic food intolerances are mainly related to a restricted capability of the gut to digest and absorb sugars and other normal dietary components. Genuine lactose intolerance is mostly due to low intestinal lactase availability but it becomes clinically manifest only with relatively large lactose loads, hence easily avoidable by forewarned patients ${ }^{19}$. Fructose, a monosaccharide present in many processed foods, has a relatively low absorptive threshold in the human small bowel and consequently fructose rich diets may produce gut symptoms in susceptible individuals by stimulating intestinal motility ${ }^{20}$. Psychological factors may influence the symptomatic response to sugar malabsorption as it has been well documented for lactose $\mathrm{e}^{21}$. Thus, subjective symptom assessment represents a potential clinical confounding influence that must be taken into consideration.

The mechanisms of abdominal symptoms developing in the context of nutrient malabsorption involve an increase in small bowel effluents entering the colon ${ }^{22}$. In turn, these molecules are fermented by colonic bacteria increasing colonic production of gas and generating osmotic and motility stimulants that induce colonic discharges and spasmodic bowel activity. There are, in addition to lactose and fructose, many incompletely absorbed carbohydrates in a normal diet that under certain circumstances may also produce colonic overload of fermentable substrates generating gas, which is poorly tolerated by IBS patients. These include the group known as FODMAP (fructans, galactosaccharides and polyols including sorbitol, mannitol and xylitol) which are naturally present in many fruits and vegetables as well as in additives such as artificial sweeteners (Figure 1). Substantial evidence has accumulated indicating that a diet limiting FODMAP components ameliorates IBS symptoms. Responders appear to harbour more saccharolytic bacterial species in 


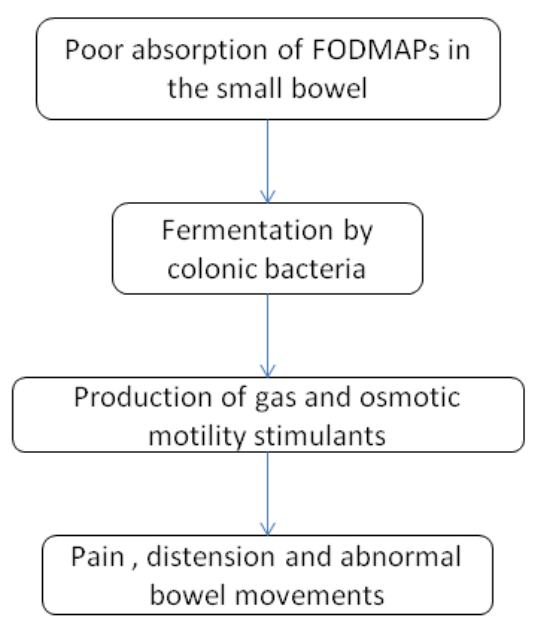

Figure 1: Mechanism of FODMAP-related IBS symptos

their microbiome ${ }^{23}$. Unfortunately, long-term sustainability of this and other highly restrictive diets is burdensome to many patients. Furthermore, it has been proposed that conventional and sensible diet restrictions may be as effective as a strict FODMAP diet although the matter is still subject to debate ${ }^{24}$. The latter approach may consist in avoiding milk products, fatty foods, drinks with artificial sweeteners or containing caffeine.

\section{Microbial ecology}

On this topic, there are two aspects that are relevant to IBS pathogenesis and management. First, there is the issue of post-infectious IBS, well documented by epidemiological follow up studies in individuals, both children and adults, ${ }^{25}$ previously affected by a bout of infectious gastroenteritis. About 10\% (figures vary) of such individuals subsequently develop symptoms compatible with $\mathrm{IBS}^{26}$. Giardiasis also represents a recognized risk factor ${ }^{27,}{ }^{28}$. Young age, psychological disturbances, prior antibiotics and other factors appear to predispose to post-infectious gastroenteritis IBS-like syndrome ${ }^{29}$. Pathogenetic factors include mucosal immune cell activation and immune cell proliferation that may enhance peripheral sensory signalling and result in visceral hypersensitivity ${ }^{30}$. Remarkably, some forms of infectious gastroenteritis in childhood, such as that caused by Salmonella species increase the probability of developing IBS in adulthood ${ }^{31}$.

A second aspect of microbial implication in IBS pathogenesis involves microbiome perturbations. To this end, two settings must be considered: excessive microbial translocation to the small bowel (small bowel bacterial overgrowth-SIBO-) and alterations in microbiome ecology. Evidence for SIBO derives primarily from breath test analysis. Unfortunately, rapid small bowel transit of the test substrate may cause an early peak signal due to early arrival of substrate into the colon that could be misinterpreted as intraluminal small bowel fermentation ${ }^{32}$. Changes in the colonic microbiome have also been implicated in IBS pathogenesis. Indeed, certain human microbiome species may influence gut function and even potentially modify gut-brain interactions. As examples, it has been observed that $F$. prausnitzii may modulate colonic hypersensitivity and Lactobacillus reuterii participates in the regulation of colonic transit ${ }^{33}$. Microbiota changes observed in postinfectious IBS include increased presence of the Bacteroidetes phylum which resembles the microbiota composition observed in diarrhea predominant IBS $^{34}$. Further research, however, will be needed to clarify these aspects, as current data are preliminary and still cannot be accepted as conclusive.

Efforts to restore microbial ecology include antibiotics, prebiotics and probiotics. Antibiotics have been proposed as treatment for presumed SIBO and also to "redress" microbiome composition. In this regard, rifaximin has been favoured based on its non-absorbability and empirical evidence of efficacy. In adults, relatively high doses of 800 to $1650 \mathrm{mg} /$ day over 10-14 days appear to improve global IBS symptomatology and IBS-related bloating. Data in children suggest that rifaximin may be particularly helpful in lactulose breath test positive patients ${ }^{35,36}$. The clinically favourable modulatory effect of rifaximin, in contrast to previously tried and unhelpful broad-spectrum antibiotics, is intriguing. It may be related to the higher bowel intraluminal concentrations achieved with rifaximin on account of its non-absorbability ${ }^{37}$. An additional factor may be a putative specific action of rifaximin on bacterial virulence and bacterial metabolic activity. In turn, these may attenuate visceral hypersensitivity and reduce hyperalgesia.

Probiotics currently enjoy great popularity among patients and many physicians on account of the intuitive appeal of its assumed mechanism of action and inherent safety (although some doubts have been raised on this latter issue). However, although experimental evidence has sometimes substantiated their antiinflammatory, antinociceptive and even their modifying effects on brain behaviour and mood, outcomes of clinical studies remain equivocal and sometimes contradictory. As Shanahan and Quigley have pointed out, ${ }^{38}$ there are many soft claims plaguing probiotics including unverified labels, gaps between research findings and marketplace claims. Also unmet label assertions on numbers and types of viable microbes as well as on shelf specifications regarding survival of packaged microbial products. Although some probiotics have been subjected to well designed and robust trials with positive outcomes, other unsuccessful trials have been reported. In children, Francavilla et $\mathrm{al}^{39}$ have shown positive responses to Lactobacillus GG and other investigators also to Lactobacillus reuterii, VSL \#3, 
Bifidobacterium infantis, brevis and longum ${ }^{40}$. Clearly, only further quality research can assert the real value of probiotics in IBS therapy.

Prebiotics are food products that favour proliferation of bacterial species that potentially diminish mucosal inflammation. Prebiotics have the theoretical drawback that they may favour gas production via intraluminal fermentation. However, recent data suggests this may be a temporary phenomenon, which delays, but does not eliminate their otherwise favourable effects ${ }^{41}$.

\section{Gut inflammation}

An inflammatory basis for IBS type symptoms has been substantiated by direct evidence of altered mucosal immune activity, presence of mucosal immune cell infiltrates, mast cell proliferation in close proximity to nerve endings, increased apical junction permeability and other features ${ }^{16,}$ 17,42 . These inflammatory responses may result from postinfectious, allergic or idiopathic gut reaction. Clinical evidence of IBS symptomatology developing in atopic individuals with or without asthmatic, cutaneous or other tissular immune inflammatory responses is mounting. Microbial-induced mucosal immune activity associated with specific bacterial species in the gut has also been reported $^{43}$.

Despite this enticing experimental background, introduction of anti-inflammatory agents to ameliorate IBS clinical manifestations has not met so far with much clinical success. Corticosteroids, mesalazine and other agents with anti-inflammatory action have not proven particularly effective ${ }^{44}$. Only the mast cell stabilizer ketotifen appears to have achieved preliminary positive results ${ }^{45}$.

\section{Intervening along the brain-gut axis}

The "two brains" theoretical model, which envisions the central brain operating in concert with the enteric brain to monitor and regulate gut function, remains imperfectly defined and only partially substantiated by experimental data. However, it is a powerful concept and potentially useful in clinical management because it provides a framework for pharmacological intervention along the central-peripheral axis ${ }^{46}$. An important additional element to take into account is the close and well established interaction between the nervous and immune systems in the gut ${ }^{47}$.

In functional conditions such as IBS, pain is a subjective experience that even if arising from nociceptive stimuli in the gut, is shaped by mechanisms acting at various strata along the brain-gut axis. Amplification and distortion of afferent signals by inflammation, sensitization and other local factors results in disturbed pain signalling. Furthermore, impaired descending pain modulation further amplifies inputs to central circuits for pain reception and modulation.
Centrally there is a key link between emotion and cognitive pain modulation that underscores the strong influence of stress and anxiety on the perception of visceral signals. Hence, abdominal pain, a hallmark of IBS, probably results from a combination of disturbed peripheral signalling and disturbed emotional pain modulation ${ }^{48}$.

IBS pain management is shifting towards agents that modulate visceral hypersensitivity exerting their actions, still poorly understood, at various sites in the gut-brain axis. In this regard, it has been pointed out that the intensity and refractoriness of the pain manifested by patients may provide some indication of the predominant mechanism of nociception. Broadly speaking, more intense and unremitting pain levels point towards a greater involvement of brain as opposed to abdominal mechanisms ${ }^{48}$. Consequently, in severe cases there is a greater need for employing neuropharmacological agents operating at a central level. In this regard, GABA blockers and antidepressants, particularly those that include inhibitory action on norepinephrine reuptake seem particularly effective. Classic tricyclic antidepressants such as amitriptyline, nortriptyline and desipramine that incorporate multiple target mechanisms may also be useful although they tend to be associated with more troublesome side effects, such as somnolence, anticholinergic systemic effects and hypotension. Dose titration is essential. Some classes of antidepressants act on specific receptors that make them particularly useful for specific complaints. For instance, the $\mathrm{H}_{1}$ receptor affinity of mirtazapine may be used to correct insomnia and to promote weight gain. Among GABA inhibitors, pregabaline has strong inhibitory effects on generalized anxiety and nociception which may be quite useful ${ }^{49}$.

The practical usefulness of neuropharmacologic agents on pain management cannot be easily separated from their effects on an underlying anxiety/ depression disorder. However, the relatively low doses of these drugs that are generally employed for IBS treatment are usually lower than customary for treatment of psychiatric conditions, unless the psychopathological component requires full use of psychotropics.

Psychological approaches including behavioural treatment have been enthusiastically put forward as a non-pharmacologic approach to correct the emotional component of functional type disorders. These include psychotherapies to alleviate stress-induced anxiety such as cognitive behavioural therapy and psychodynamic therapy. Hypnotherapy performed by a well trained therapist may help the child relax into a trance-like state. These formally conducted psychological treatments have been reported to achieve substantial success and are increasingly undertaken today. However, clinicians should be aware of the high placebo response rate observed in most controlled 
trials of IBS and related conditions (30-70\%). The more structured psychotherapeutic approaches, in addition, are time consuming and their long-term effectiveness remains unproven.

\section{Acting on intestinal fluid and motor dynamics}

At a peripheral level, drugs acting on motor and/or secretory function have long been employed to ameliorate pain and also the characteristic bowel movement pattern in IBS, be it constipation and/or diarrhea that may, by interfering with orderly propulsion, cause pain. This group of agents includes classical antispasmodics, antidiarrheals and laxatives of various types. Time sanctioned use of such agents, which attests to their popularity, does not imply proven clinical efficacy. In particular, classic antispasmodics with anticholinergic action (hence, with unpleasant side effects) and non-anticholinergic antispasmodics (primarily calcium channel blockers) such as mebeverine and otilonium bromide, although widely used, have only gathered weak scientific evidence for their clinical effectiveness.

Conventional antidiarrheal agents such as loperamide are commonly employed, but, in IBS-associated diarrhea they are difficult to modulate and show a propensity to precipitate constipation, that disquiets the patient who usually expects a more stable regulatory action on bowel movements?

Diarrhea in IBS may also be caused or aggravated by bile acid malabsorption with overspillage into the colon where these molecules, particularly in their unconjugated form (as by-products of bacterial hydrolysis), irritate the colonic mucosa and induce water exorption ${ }^{50}$. Overspillage of bile acids may be the result of either abnormally accelerated small bowel transit, ileal mucosal impairment with reduced ileal bile acid reabsorption or liver overproduction. Regardless of the specific mechanism involved, oral bile acid sequestrants acting intraluminally such as cholestyramine and the more palatable product colesevelam may help control diarrhea ${ }^{51}$. Careful dose titration of these agents is a requirement to prevent an unwarranted shift towards constipation and bloating.

Eluxadoline is a complex opiod receptor activator that has proven useful as antidiarrheal and antinociceptive agent for IBS- $\mathrm{D}^{52}$. Its effects are more subtle than the traditional antidiarrheal agent loperamide, but it has shown a disturbing, although unusual, propensity to cause pancreatitis, albeit mostly in cholecystectomized patients.

The therapeutic effect of conventional laxatives may be difficult to modulate in children. PEG solutions are the preferred agent. Newer, potentially useful agents are coming along. Linaclotide, an agonist of enteric guanylate cyclase, and lubiprostone, a prostaglandin $\mathrm{E}$ analogue, both promote water exorption into the gut. In addition, they have putative antinociceptive effects that make them suitable for the management of IBS-C. Unfortunately, although quite useful in adults, so far, neither of these two agents are approved for therapeutic purposes in children (and even some negative effects have been reported for lubiprostone in this young age group $)^{53}$.

\section{Acting on parental roles and influencers}

Family environment may act positively or negatively on children's expression of IBS symptomatology. It appears that parenteral attitudes, modelling of symptoms, coping methods, psychological traits and family exposure to stress have potential influence on gastrointestinal and nongastrointestinal symptom reporting by children ${ }^{54}$. Thus, recruiting parents, and maybe other family members, to help modify the child's illness behaviour and symptom impact on daily activities has some theoretical foundation. However, studies to substantiate the therapeutic benefit of interventions through relatives have yet to be conducted.

\section{References}

1. Camilleri M, Di Lorenzo C. Brain-gut axis: from basic understanding to treatment of IBS and related disorders. J Pediatr Gastroenterol Nutr. 2012; 54: 446-53.

2. Self MM, Czyzewski DI, Chumpitazi BP, et al. Subtypes of irritable bowel syndrome in children and adolescents. Clin Gastroenterol Hepatol. 2014; 12: 1468-73.

3. Giannetti E, de'Angelis G, Turco R, et al. Subtypes of irritable bowel syndrome in children: prevalence at diagnosis and at follow-up. J Pediatr. 2014; 164: 1099-1103 e1.

4. Saps M, Velasco-Benitez CA, Bloom PJJ, et al. Prospective Study of Gastrointestinal Symptoms in School Children of South America. J Pediatr Gastroenterol Nutr. 2018; 66: 391-394.

5. Malagelada JR, Malagelada C. Mechanism-Oriented Therapy of Irritable Bowel Syndrome. Advances in Therapy. 2016; 33: 877-893.

6. Hyams JS, Burke G, Davis PM, et al. Abdominal pain and irritable bowel syndrome in adolescents: a community-based study. J Pediatr. 1996; 129: 220-6.

7. Sandhu BK, Paul SP. Irritable bowel syndrome in children: pathogenesis, diagnosis and evidence-based treatment. World J Gastroenterol. 2014; 20: 6013-23.

8. Hoekman DR, Rutten JM, Vlieger AM, et al. Annual Costs of Care for Pediatric Irritable Bowel Syndrome, Functional Abdominal Pain, and Functional Abdominal Pain Syndrome. J Pediatr. 2015; 167: 1103-8 e2.

9. Quigley EM, Shanahan F. The language of medicine: words as servants and scoundrels. Clin Med (Lond). 2009; 9: 131-135.

10. Philips EM, Peeters B, Teeuw AH, et al. Stressful Life Events in Children With Functional Defecation Disorders. J Pediatr Gastroenterol Nutr. 2015; 61: 384-92.

11. Chogle A, Mintjens S, Saps M. Pediatric IBS: an overview on pathophysiology, diagnosis and treatment. Pediatr Ann. 2014; 43: e76-82.

12. Chang L, Berman S, Mayer EA, et al. Brain responses to visceral and somatic stimuli in patients with irritable bowel syndrome with and without fibromyalgia. Am J Gastroenterol. 2003; 98: 1354-61.

13. Seino S, Watanabe S, Ito N, et al. Enhanced auditory brainstem 
response and parental bonding style in children with gastrointestinal symptoms. PLoS One. 2012; 7: e32913.

14. Giannetti E, Maglione M, Sciorio E, et al. Do Children Just Grow Out of Irritable Bowel Syndrome. J Pediatr. 2017; 183: 122-126.e1.

15. Ross SE. "Memes" as infectious agents in psychosomatic illness. Ann Intern Med. 1999; 131: 867-71.

16. Matricon J, Meleine M, Gelot A, et al. Review article: Associations between immune activation, intestinal permeability and the irritable bowel syndrome. Aliment Pharmacol Ther. 2012; 36: 1009-31.

17. Cremon C, Gargano L, Morselli-Labate AM, et al. Mucosal immune activation in irritable bowel syndrome: gender-dependence and association with digestive symptoms. Am J Gastroenterol. 2009; 104: 392-400.

18. Elli L, Rossi V, Conte D, et al. Increased Mercury Levels in Patients with Celiac Disease following a Gluten-Free Regimen. Gastroenterol Res Pract. 2015; 2015: 953042.

19. Itan $\mathrm{Y}$, Jones $\mathrm{BL}$, Ingram $\mathrm{CJ}$, et al. A worldwide correlation of lactase persistence phenotype and genotypes. BMC Evol Biol. 2010; 10: 36.

20. Madsen JL, Linnet J, Rumessen JJ. Effect of nonabsorbed amounts of a fructose-sorbitol mixture on small intestinal transit in healthy volunteers. Dig Dis Sci. 2006; 51: 147-53.

21. Casellas F, Aparici A, Casaus M, et al. Subjective perception of lactose intolerance does not always indicate lactose malabsorption. Clin Gastroenterol Hepatol. 2010; 8: 581-6.

22. Murray K, Wilkinson-Smith V, Hoad C, et al. Differential effects of FODMAPs (fermentable oligo-, di-, mono-saccharides and polyols) on small and large intestinal contents in healthy subjects shown by MRI. Am J Gastroenterol. 2014; 109: 110-9.

23. Chumpitazi BP, Cope JL, Hollister EB, et al. Randomised clinical trial: gut microbiome biomarkers are associated with clinical response to a low FODMAP diet in children with the irritable bowel syndrome. Aliment Pharmacol Ther. 2015; 42: 418-27.

24. Böhn L, Störsrud S, Liljebo T, et al. Diet low in FODMAPs reduces symptoms of irritable bowel syndrome as well as traditional dietary advice: a randomized controlled trial. Gastroenterology. 2015; 149: 1399-1407.e2.

25. Thabane M, Simunovic M, Akhtar-Danesh N, et al. An outbreak of acute bacterial gastroenteritis is associated with an increased incidence of irritable bowel syndrome in children. Am J Gastroenterol. 2010; 105: 933-9.

26. Downs IA, Aroniadis OC, Kelly L, et al. Postinfection Irritable Bowel Syndrome: The Links Between Gastroenteritis, Inflammation, the Microbiome, and Functional Disease. J Clin Gastroenterol. 2017; 51: 869-877.

27. Hanevik K, Dizdar V, Langeland N, et al. Development of functional gastrointestinal disorders after Giardia lamblia infection. BMC Gastroenterol. 2009; 9: 27.

28. Nakao JH, Collier SA, Gargano JW. Giardiasis and Subsequent Irritable Bowel Syndrome: A Longitudinal Cohort Study Using Health Insurance Data. J Infect Dis. 2017; 215: 798-805.

29. Schwille-Kiuntke J, Enck P, Zendler C, et al. Postinfectious irritable bowel syndrome: follow-up of a patient cohort of confirmed cases of bacterial infection with Salmonella or Campylobacter. Neurogastroenterol Motil. 2011; 23: e479-88.

30. Spreadbury I, Ochoa-Cortes F, Ibeakanma C, et al. Concurrent psychological stress and infectious colitis is key to sustaining enhanced peripheral sensory signaling. Neurogastroenterol Motil. 2015; 27: 347-55.

31. Cremon C, Stanghellini V, Pallotti F, et al. Salmonella gastroenteritis during childhood is a risk factor for irritable bowel syndrome in adulthood. Gastroenterology. 2014; 147: 69-77.
32. Yu D, Cheeseman F, Vanner S. Combined oro-caecal scintigraphy and lactulose hydrogen breath testing demonstrate that breath testing detects oro-caecal transit, not small intestinal bacterial overgrowth in patients with IBS. Gut. 2011; 60: 334-40.

33. Miquel S, Martín R, Lashermes A, et al. Anti-nociceptive effect of Faecalibacterium prausnitzii in non-inflammatory IBS-like models. Sci Rep. 2016; 6: 19399.

34. Jalanka-Tuovinen J, Salojarvi J, Salonen A, et al. Faecal microbiota composition and host-microbe cross-talk following gastroenteritis and in postinfectious irritable bowel syndrome. Gut. 2014; 63: 173745.

35. Scarpellini E, Giorgio V, Gabrielli M, et al. Rifaximin treatment for small intestinal bacterial overgrowth in children with irritable bowel syndrome. Eur Rev Med Pharmacol Sci. 2013; 17: 1314-20.

36. Collins BS, Lin HC. Double-blind, placebo-controlled antibiotic treatment study of small intestinal bacterial overgrowth in children with chronic abdominal pain. J Pediatr Gastroenterol Nutr. 2011; 52: 382-6.

37. Jiang ZD, Ke S, Palazzini E, et al. In vitro activity and fecal concentration of rifaximin after oral administration. Antimicrob Agents Chemother 2000; 44: 2205-6.

38. Shanahan F, Quigley EM. Manipulation of the microbiota for treatment of IBS and IBD-challenges and controversies. Gastroenterology. 2014; 146: $1554-63$.

39. Francavilla R, Miniello V, Magista AM, et al. A randomized controlled trial of Lactobacillus GG in children with functional abdominal pain. Pediatrics. 2010; 126: e1445-52.

40. Giannetti E, Staiano A. Probiotics for Irritable Bowel Syndrome: Clinical Data in Children. J Pediatr Gastroenterol Nutr. 2016; 63 Suppl 1: $\mathrm{S} 25-6$.

41. Huaman JW, Mego M, Manichanh C, et al. Effects of Prebiotics vs a Diet Low in Fodmaps in Patients with Functional Gut Disorder. Gastroenterology. 2018; Jun 28 [Epub ahead of print].

42. O'Malley D. Immunomodulation of enteric neural function in irritable bowel syndrome. World J Gastroenterol. 2015; 21: 7362-6.

43. Powell N, Walker MM, Talley NJ. The mucosal immune system: master regulator of bidirectional gut-brain communications. Nat Rev Gastroenterol Hepatol. 2017; 14: 143-159.

44. Törnblom H, Simrén M. In search for a disease-modifying treatment in irritable bowel syndrome. Gut. 2016; 65: 2-3.

45. Klooker TK, Braak B, Koopman KE, et al. The mast cell stabiliser ketotifen decreases visceral hypersensitivity and improves intestinal symptoms in patients with irritable bowel syndrome. Gut. 2010; 59: 1213-21.

46. Koloski NA, Jones M, Kalantar J, et al. The brain--gut pathway in functional gastrointestinal disorders is bidirectional: a 12-year prospective population-based study. Gut. 2012; 61: 1284-90.

47. Ghia JE, Blennerhassett P, Collins SM. Impaired parasympathetic function increases susceptibility to inflammatory bowel disease in a mouse model of depression. J Clin Invest. 2008; 118: 2209-18.

48. Drossman DA. Redux: do little bellyachers grow up to become big bellyachers. Clin Gastroenterol Hepatol. 2014; 12: 2033-6.

49. Saps M, Miranda A. Gastrointestinal Pharmacology. Handb Exp Pharmacol. 2017; 239: 147-176.

50. Bajor A, Törnblom H, Rudling $M$, et al. Increased colonic bile acid exposure: a relevant factor for symptoms and treatment in IBS. Gut. 2015; 64: 84-92.

51. Camilleri M, Acosta A, Busciglio I, et al. Effect of colesevelam on faecal bile acids and bowel functions in diarrhoea-predominant irritable bowel syndrome. Aliment Pharmacol Ther. 2015; 41: 438-48. 
52. Wade PR, Palmer JM, McKenney S, et al. Modulation of gastrointestinal function by MuDelta, a mixed $\mu$ opioid receptor agonist/ $\mu$ opioid receptor antagonist. Br J Pharmacol. 2012; 167: 1111-25.

53. Hyman PE, Di Lorenzo C, Prestridge LL, et al. Lubiprostone for the treatment of functional constipation in children. J Pediatr Gastroenterol Nutr. 2014; 58: 283-91.

54. van Tilburg MA, Levy RL, Walker LS, et al. Psychosocial mechanisms for the transmission of somatic symptoms from parents to children. World J Gastroenterol. 2015; 21: 5532-41. 